\title{
Prevalence and patterns of tooth agenesis among patients aged 12-22 years: A retrospective study
}

\author{
Basak Kiziltan Eliacik (1) \\ Cafer Atas \\ Gunseli Guven Polat
}

Department of Pediatric Dentistry, Hamidiye Faculty of Dental Medicine, University of Health Sciences, lstanbul, Turkey
Objective: This study aimed to establish the prevalence and patterns of nonsyndromic tooth agenesis in patients referred to a tertiary health care facility. Methods: The intraoral records and panoramic radiographs of 9,874 patients aged 12-22 years were evaluated. The study group included 716 patients (371 male, 345 female) with non-syndromic agenesis of at least one tooth (except the third molars). The study data were assessed using descriptive statistics, chisquare test, and Mann-Whitney $\mathrm{U}$ test, while patterns were evaluated using a tooth agenesis code (TAC) tool. Results: A total of 1,627 congenitally missing teeth, were found in patients with non-syndromic tooth agenesis, with an average of 2.27 missing teeth per patient. The prevalence of tooth agenesis was $7.25 \%$, and the most commonly missing teeth were the left mandibular second premolars $(10.17 \%)$. The age group comparison revealed no significant difference in the median number of missing teeth per patient according to the cutoff values for ages between 12 and 22 years. When the missing teeth were examined separately according to quadrants, 114 different tooth agenesis patterns (upper right quadrant $=28$, upper left quadrant $=27$, lower left quadrant $=31$, and lower right quadrant $=28$ ) were identified, and 81 of these patterns appeared only once. Conclusions: This study highlights the benefits of applying the TAC tool in a large sample population. The application of the TAC tool in such studies will enable the development of template treatment plans by determining homogenous patterns of tooth agenesis in certain populations.

[Korean J Orthod 2021;51(5):355-362]

Key words: Panoramic radiography, Prevalence, Hypodontia, Retrospective study

Received January 25, 2021; Revised March 18, 2021; Accepted March 29, 2021.

Corresponding author: Basak Kiziltan Eliacik.

Assistant Professor, Department of Pediatric Dentistry, Hamidiye Faculty of Dental Medicine, University of Health Sciences, Mekteb-i Tıbbiye-i Şahane (Haydarpaşa) Külliyesi Selimiye Mah. Tıbbiye Cad. No: 3834668 Üsküdar, İstanbul, Turkey.

Tel+905300865217 e-mail basak.eliacik@sbu.edu.tr

How to cite this article: Kiziltan Eliacik B, Atas C, Guven Polat G. Prevalence and patterns of tooth agenesis among patients aged 12-22 years: A retrospective study. Korean J Orthod 2021;51:355-362.

(C) 2021 The Korean Association of Orthodontists.

This is an Open Access article distributed under the terms of the Creative Commons Attribution Non-Commercial License (http://creativecommons.org/licenses/by-nc/4.0) which permits unrestricted non-commercial use, distribution, and reproduction in any medium, provided the original work is properly cited. 


\section{INTRODUCTION}

Development of human dentition is a complex process that involves both genetic and molecular factors.' Any defect in this process affects various aspects of dental development (i.e., number, form, shape, or structure). One such defect with a high incidence is tooth agenesis, which is a congenital anomaly characterized by the absence of one or more teeth in the jaws. The etiology of tooth agenesis may involve genetic mutations as well as epigenetic and environmental factors.

The absence of 1-6 teeth is called hypodontia, whose incidence is higher than that of other congenital dental anomalies. ${ }^{2-4}$ Various studies have investigated the prevalence of tooth agenesis in different populations and geographical regions (e.g., Japan, Australia, Iran, Turkey, Romania, Israel, and Norway). The variance in tooth agenesis across populations is believed to result from differences in sex, age, and race, as well as the use of different research methods. The absence of $\geq 6$ teeth is referred to as severe hypodontia (oligodontia). Although oligodontia is usually accompanied by genetic syndromes (ectodermal dysplasia, Klinefelter syndrome, incontinentia pigmenti, etc.), patients with non-syndromic oligodontia are also encountered at clinics. ${ }^{5}$ The prevalence of oligodontia is reported to range from $0.08 \%$ to $0.16 \%$ in various populations. ${ }^{6-8}$ Previous studies on tooth agenesis have also reported that the most commonly missing teeth are the mandibular second premolars, lateral incisors, and maxillary second premolars. ${ }^{2,4,5,9}$

Since the non-development of tooth germs causes many esthetic and functional problems, including the misplacement of teeth, periodontal damage, or inadequate bone height in the upper and lower jaws, the diagnosis should be established without delay in such patients, and their treatment should be planned using a multidisciplinary approach. ${ }^{10}$ From this perspective, potential future dental problems of children can be minimized through the diagnosis of missing teeth by pediatric dentists at their first oral examination, by performing regular radiographic examinations starting from the very early ages, and by planning the treatment accordingly.

Research indicates that the rates of tooth agenesis are higher today than in the past. ${ }^{11}$ Studies conducted in the Turkish population revealed that the prevalence of tooth agenesis ranged between $1.77 \%$ and $7.54 \% .{ }^{12,13}$ A study by Kirzioğlu et al. ${ }^{14}$ identified agenesis-the absence of tooth germs-in 192 individuals aged 1-18 years old. However, in this age group, the identification of germs using radiographs will not provide accurate results. Therefore, that study has limitations in terms of both the age range and the number of radiographs examined. The number of radiographs included in the studies by Gökkaya et al. ${ }^{15}$ and Sisman et al. ${ }^{11}$ was, in turn, consid- erably less than that included in the present study. Furthermore, none of the studies conducted in the Turkish population identified agenesis patterns by using tooth agenesis code (TAC) analysis.

Therefore, the present study aimed to investigate the prevalence of non-syndromic tooth agenesis and to identify patterns of hypodontia and severe hypodontia in a large sample of the Turkish population by using a classification method created by van Wijk and Tan, ${ }^{16}$ which considered the different phenotypes of this condition.

\section{MATERIALS AND METHODS}

This retrospective, observational, epidemiological study included 9,874 patients aged 12-22 years old $(4,127$ male, 5,747 female) who were referred to a tertiary health care facility between 2016 and 2020. The study protocol was evaluated and approved by the University of Health Sciences Scientific Research Ethics Committee (46418926-050.03.04/19/143). The radiographs were acquired using an Orthopantomograph ${ }^{\circledR}$ OP200 (Instrumentarium Dental, Tuusula, Finland) device and processed using its inbuilt software. Panoramic radiographs and anamnestic data of the patients were evaluated, and the diagnosis of absence of a tooth and/or teeth (nonsyndromic hypodontia or non-syndromic oligodontia) was established. Panoramic radiographs were evaluated by two different, experienced, and previously trained pedodontists at different times, by using the visual method and an identical protocol. To avoid any examination bias, all panoramic radiographs were reexamined by the same investigators after an interval of 15 days. The exclusion criteria were as follows: an unclear identification of tooth agenesis on panoramic radiographs, in which case the physicians were hesitant about the presence of the permanent dental germ; the presence of developmental diseases (ectodermal dysplasia, cleft lip-cleft palate, etc.); absence of tooth because of extraction for any reason (trauma, periodontal, or pathological); and previous orthodontic treatment. The third molars were also excluded to avoid miscounting the missing teeth considering the mineralization of these teeth. A tooth was considered missing when any sign of any stage of mineralization of the dental crown could not be determined during the examination of the panoramic radiographs.

The patterns of tooth agenesis among the study patients were evaluated using the TAC tool (http://www. toothagenesiscode.com; last access on December 1, 2020) based on a binary code developed by van Wijk and Tan. ${ }^{16}$ The TAC methodology aims to obtain information about which teeth are missing simultaneously and to answer specific research questions (e.g., whether 
dominant patterns of tooth agenesis can be identified), which can increase the researchers' understanding of tooth agenesis and contribute to the selection of patients for future genetic studies. Human dentition includes 8 teeth in each quadrant, which are numbered from 1 to 8 according to the Federation Dentaire International system. ${ }^{16}$ The scoring system of van Wijk and Tan is dichotomized as the presence (0) or absence (1) of each tooth. A certain value is calculated for each missing tooth, and the sum of these values represents a specific value called the TAC, which describes the pattern of tooth agenesis for the entire mouth (Table 1). According to the TAC, a quadrant without tooth agenesis has a value of 0 , while a quadrant with complete tooth agenesis has a value of 255. For example, when $\mathrm{TAC}=024.000 .000 .024$, the number 024 refers to tooth agenesis in the upper right dental quadrant $(14,15)$; the second number 024 represents tooth agenesis in the lower right dental quadrant $(44,45)$; and the number 000 indicates no tooth agenesis in the upper left and lower left dental quadrants. The number 024 (in the upper right dental quadrant) is the sum of 16 (for the missing second premolar) and 8 (for the missing first premolar).

All statistical analyses were conducted using IBM SPSS Statistics for Windows, Version 22.0 (IBM Corp., Armonk, NY, USA). Descriptive statistics were used to present the patient characteristics. Intraexaminer agreement was evaluated using the percentage of different patterns identified in the two repeated assessments. The number of missing teeth and prevalence (\%) of tooth agenesis for each tooth type in the maxillary and mandibular arches, excluding the third molars, were calculated for each patient. Patients were then grouped according to sex and age. The distribution of missing teeth was assessed using the Shapiro-Wilk test. No group showed normal distribution. The relationship between the number of missing teeth and sex was analyzed using the chisquare test. Medians were calculated and compared between certain age groups from 12 to 22 years old. The nonparametric Mann-Whitney $U$ test was used, and this statistical comparison was repeated for the 11 different cutoff ages. The significance level used for analysis was
$5 \%(p=0.05)$

\section{RESULTS}

Both intraexaminer and interexaminer agreements in this study were near perfect (99\% and 97.5\%, respectively). A total of 1,627 (range, 1-8) congenitally missing teeth, excluding the third molars, were found in 716 patients (371 male, 345 female) with non-syndromic tooth agenesis, with an average of 2.27 missing teeth per patient in the permanent dentition after a careful check of 9,874 (4,127 male, 5,747 female) patient files. The prevalence of tooth agenesis was $7.25 \%$, and in most cases, 2 teeth were missing $(n=306 ; 42.7 \%)$. Male patients had 831 missing teeth, with an average of 2.24 missing teeth per patient; female patients had 796 missing teeth, with an average of 2.31 missing teeth per patient. The distribution of the samples according to the number of missing teeth is shown in Table 2. The difference in the prevalence of missing teeth per patient

Table 2. Descriptive statistics of the whole sample and prevalence rates of tooth agenesis in the present study

\begin{tabular}{lc}
\hline \multicolumn{1}{c}{ Variable } & Value \\
\hline Total & $9,874(100)$ \\
Sex & \\
Male & $4,127(41.8)$ \\
Female & $5,747(58.2)$ \\
Teeth agenesis & \\
Yes & $716(7.2)$ \\
No & $9,158(92.8)$ \\
Number of missing teeth & \\
1 & $232(32.4)$ \\
2 & $306(42.7)$ \\
3 & $46(6.4)$ \\
4 & $65(9.1)$ \\
$\geq 5$ & $67(9.4)$ \\
\hline
\end{tabular}

Values are presented as number (\%).

Table 1. Tooth agenesis code (TAC) system

\begin{tabular}{|c|c|c|c|c|c|c|c|c|c|c|c|c|c|c|c|c|c|}
\hline & & \multicolumn{6}{|c|}{ Upper right quadrant (q1) } & \multicolumn{10}{|c|}{ Upper left quadrant (q2) } \\
\hline A & 18 & 17 & 16 & 15 & 14 & 13 & 12 & 11 & 21 & 22 & 23 & 24 & 25 & 26 & 27 & 28 & $\mathrm{~A}$ \\
\hline B & 128 & 64 & 32 & 16 & 8 & 4 & 2 & 1 & 1 & 2 & 4 & 8 & 16 & 32 & 64 & 128 & $\mathrm{~B}$ \\
\hline A & 48 & 47 & 46 & 45 & 44 & 43 & 42 & 41 & 31 & 32 & 33 & 34 & 35 & 36 & 37 & 38 & $\mathrm{~A}$ \\
\hline & & \multicolumn{6}{|c|}{ Lower right quadrant (q4) } & \multicolumn{10}{|c|}{ Lower left quadrant (q3) } \\
\hline
\end{tabular}

A shows Federation Dentaire Internationale tooth numbering system that is used in the dental field. B refers to as values of the TAC that can be described any pattern of tooth agenesis in numeric style. 
between the sexes was not statistically significant ( $p=$ 0.27). No significant difference was observed in the median numbers of missing teeth per patient between the younger and older age groups according to the different cutoff points between 12 and 22 years of age (Table 3). The distribution of missing teeth across quadrants and tooth numbers is shown in Table 4. When the missing teeth were examined separately according to the quadrants, 114 different tooth agenesis patterns (upper right quadrant $[q 1]=28$, upper left quadrant [q2] $=27$, lower left quadrant $[\mathrm{q} 3]=31$, and lower right quadrant $[\mathrm{q} 4]=$ 28) were identified, and 81 of these patterns appeared only once. The top five ranked patterns in the different quadrants are shown in Table 5. In female patients, the prevalence of tooth agenesis, i.e., 1, 2, 3, 4, and $\geq 5$ missing teeth, was $49.6 \%, 55.6 \%, 45.7 \%, 52.3 \%$, and $41.7 \%$, respectively. When analyzed on the basis of quadrants, the most common pattern in the maxilla and mandible was agenesis of both the premolars and lateral incisor; however, the absence of all teeth except the maxillary central incisor was also detected (Table 5). Appendix presents the distribution of missing teeth according to sex and tooth type. The most common missing teeth were the left mandibular second premolar, right mandibular second premolar, and right maxillary second premolar.

Appendix is available at https://doi.org/10.4041/ kjod.2021.51.5.355.

\section{DISCUSSION}

Tooth agenesis or hypodontia is a developmental anomaly that is currently very common. ${ }^{17-19}$ The absence of teeth in humans leads to many problems in terms of esthetics, functionality, and phonation. ${ }^{20,21}$ Early diagnosis of these conditions is important because delayed treatment can impose a financial burden owing to the reduced number of available late treatment alternatives.

Therefore, the prevalence of tooth agenesis has been investigated in many populations and is still a subject of

Table 3. Comparison of the mean numbers of missing teeth per person according to different minimum cutoff ages

\begin{tabular}{ccccccc}
\hline \multirow{2}{*}{ Cutoff age (yr) } & \multicolumn{2}{c}{ C Cutoff age } & \multicolumn{2}{c}{$\geq$ Cutoff age } & \multirow{2}{*}{ Difference } & \multirow{2}{*}{-value } \\
\cline { 2 - 4 } & $\mathbf{n}$ & Mean & $\mathbf{n}$ & Mean & & \\
\hline 12 & 285 & 2.02 & 431 & 1.99 & -0.03 & 0.767 \\
13 & 350 & 1.98 & 366 & 2.02 & 0.04 & 0.590 \\
14 & 388 & 1.99 & 328 & 2.01 & 0.02 & 0.788 \\
15 & 478 & 1.98 & 238 & 2.05 & 0.07 & 0.384 \\
16 & 559 & 2.01 & 157 & 1.98 & -0.03 & 0.855 \\
17 & 635 & 1.99 & 81 & 2.18 & 0.19 & 0.204 \\
18 & 660 & 1.98 & 56 & 2.23 & 0.25 & 0.198 \\
19 & 678 & 1.99 & 38 & 2.15 & 0.16 & 0.355 \\
20 & 699 & 2.01 & 17 & 1.94 & -0.07 & 0.791 \\
21 & 706 & 2.01 & 10 & 1.90 & -0.11 & 0.742 \\
22 & 712 & 2.00 & 4 & 2.25 & 0.25 & 0.637 \\
\hline
\end{tabular}

Mann-Whitney $U$ test was performed.

Table 4. Distribution of missing teeth across quadrants and tooth numbers

\begin{tabular}{lccccc}
\hline \multicolumn{1}{c}{ Tooth } & Upper right & Upper left & Lower left & Lower right & Total \\
\hline Central incisor & 0 & 0 & 64 & 41 & 105 \\
Lateral incisor & 71 & 90 & 71 & 80 & 312 \\
Canine & 58 & 48 & 22 & 46 & 174 \\
First premolar & 83 & 84 & 91 & 100 & 358 \\
Second premolar & 137 & 123 & 166 & 148 & 574 \\
First molar & 11 & 54 & 3 & 5 & 85 \\
Second molar & 4 & 7 & 422 & 435 & 15 \\
Total & 364 & 406 & & 527 \\
\hline
\end{tabular}




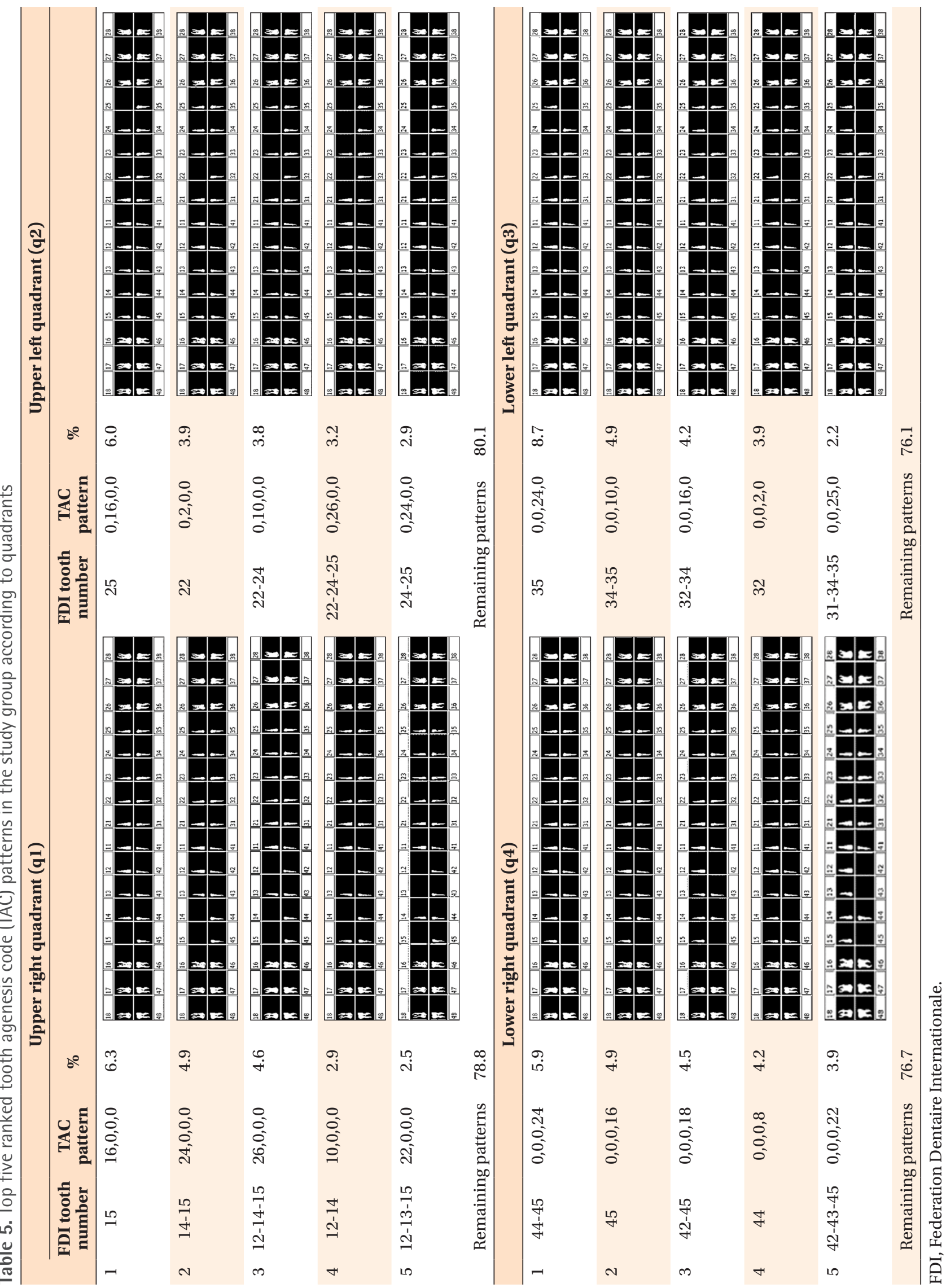


active research. ${ }^{19,22-28}$ Some of the previous studies (that included a small number of patients or included patients visiting orthodontic clinics) achieved results upon using orthodontic models and intraoral photographs in addition to radiography and anamnestic data. ${ }^{5,22,28,29}$ The present study involved screening a large number of patient radiographs at a hospital with a very high volume of patients in lstanbul. Although this sample represented only a small proportion of the Turkish population, it targeted a high number of patients $(9,874$ in total) and established the prevalence of tooth agenesis by examining only radiographs and anamnestic data. The rate of tooth agenesis has been reported to range from $1 \%$ to $11 \%$ across various populations, ${ }^{2,5,9,17,30}$ and in studies conducted in the Turkish population, this rate was found to range between $1.77 \%$ and $7.54 \% .{ }^{11-13,15}$ Gökkaya et al. ${ }^{15}$ examined the radiographs of 1,236 individuals in an age range similar to that used in the present study and established a tooth agenesis rate of 7\% $(n=82)$. In another study, Sisman et al. ${ }^{11}$ identified tooth agenesis in $182(7.54 \%)$ of the 2,413 patients examined. Similar to these studies conducted in the Turkish population, the present study established a tooth agenesis rate of 7.25\%.

Many studies that examined sex differences in the prevalence of tooth agenesis reported no statistically significant differences. Among these, the study by Endo et $\mathrm{al}^{2}{ }^{2}$ examined the data of 3,358 children aged 5-15 years old, who visited an orthodontic clinic, and detected tooth agenesis in 286 children. When the sex distribution of these children was evaluated, the authors established that 9.3\% (177) of female and 7.5\% (109) of male patients had tooth agenesis. Despite the numerical difference, no statistical difference was found. In contrast, the study by Bozga et al. ${ }^{26}$ included patients in a wider age group (6-41 years) and assessed 518 patients who visited an orthodontic clinic. The authors found the agenesis rate in 35 patients with tooth agenesis to be $7.29 \%$ and $6.31 \%$ in male and female patients, respectively. In a study of the Turkish population by Gökkaya et al., the prevalence of tooth agenesis was found to be $9 \%$ in girls and 5\% in boys, and it established a significant sex difference in tooth agenesis. Similar to the study by Gökkaya et al., ${ }^{15}$ Sisman et al. ${ }^{11}$ established a higher rate of tooth agenesis in girls (8.9\%) than in boys $(6.54 \%)$. However, these two studies were limited by their insufficient sample size when compared to that of the present study, which examined panoramic radiographs of 9,874 patients. The present study also examined the sex distribution of patients with tooth agenesis and found the incidence of tooth agenesis to be $9 \%$ and $6 \%$ in male and female patients, respectively, but without any statistically significant difference in the prevalence of missing teeth per patient. In contrast to other studies, but similar to that of Bozga et al., ${ }^{26}$ the present study established a higher prevalence of tooth agenesis among male patients.

The study by Endo et al., ${ }^{2}$ which indicated that the number of missing teeth varied between 1 and 21 among children with non-syndromic tooth agenesis, detected 1 or 2 teeth missing in $76.3 \%$ of cases, while it detected $\geq 5$ teeth missing in $10.1 \%$ of cases. A more recent study by Souza-Silva et al. ${ }^{17}$ reviewed 2,239 panoramic radiographs acquired at a radiology center and reported that 1 or 2 teeth were missing in $86.7 \%$ of 68 cases with non-syndromic tooth agenesis. The authors further reported the rate of $\geq 5$ teeth missing to be $3 \%$. In one of the most extensive multicenter studies on tooth agenesis performed to date, $\mathrm{Arai}^{5}$ investigated tooth agenesis in Japanese orthodontic patients and identified hypodontia and oligodontia in $6.61 \%$ and $0.51 \%$, respectively. In the present study, the ratios of patients with $<5$ and $\geq 5$ teeth missing on radiographs compared to those of all the patients in the study were $6.57 \%$ and $0.68 \%$, respectively, and these were consistent with those reported by Arai. ${ }^{5}$ These findings suggest that the higher the number of study participants, the closer to reality the results are in studies aimed at determining the incidence of any anomaly. Accordingly, the present study that assessed a higher number of radiographs identified a higher number of patients with $\geq$ 5 teeth missing, which was in agreement with the number reported by Arai, ${ }^{5}$ but lower than those reported by Souza-Silva et al. ${ }^{17}$ and Endo et al. ${ }^{2}$

Studies have reported that the exclusion of the missing third molar would increase the reliability of research, since the total number of patients with hypodontia increases by up to $27-30.64 \%$ when the third molar is accepted as missing. ${ }^{4,5,9,17}$ Therefore, we excluded the third molar in the present study.

The most commonly reported missing teeth are the second premolars, lateral incisors, and first premolars. ${ }^{2,530}$ Dang et al. ${ }^{30}$ and Endo et al. ${ }^{2}$ found the most common missing teeth to be the mandibular second premolars and maxillary lateral incisors, respectively. In the study by Endo et al., ${ }^{2}$ the left mandibular second premolars (10.71-13.1\%) had the highest incidence of agenesis, while the maxillary central incisors had no agenesis and the second molars had the least common agenesis. In contrast, $\mathrm{Arai}^{5}$ identified the mandibular and maxillary second premolars as the most commonly missing teeth. Similar to the study by Arai, ${ }^{5}$ the present study found the second premolars, first premolars, and lateral incisors to be the most commonly missing teeth in order of frequency. In addition, the present study established that the percentage of missing second molars in all dental quadrants was the lowest, and that agenesis of the maxillary central incisors was absent, which is consistent with the findings of previous studies. The prevalence of 
tooth agenesis and the distribution of missing teeth in this study group are also consistent with those of previous studies.

This study provides some enlightening information about the use of the TAC tool in a large sample population. Including a large number of individuals for data analysis increases the reliability and accuracy of the information obtained. The TAC tool has an advantage over graphical notation because it helps detect homogenous specific patterns of tooth agenesis and generates valuable information capable of directing future epidemiological and genetic studies on tooth agenesis by aiding in patient selection. The most common patterns among patients with hypodontia were successfully identified using the TAC tool in the Turkish population.

To our knowledge, this is one of the few studies using the TAC methodology and the first to be conducted in the Turkish population. Nevertheless, the present study has some limitations. As the study center is a tertiary health care facility, children and young adults are more frequently referred to this facility for treatment, and this could be a potential reason for the high prevalence of tooth agenesis in the current study population. Further, this was a single-center study, and repeating this research at central hospitals in other parts of Turkey would ensure the identification of the nationwide tooth agenesis rates and would considerably improve our knowledge of hypodontia. Moreover, there is a high possibility of tooth agenesis in craniofacial abnormalities and in various syndromes, which were not included in the present study. In order to establish patterns, detecting tooth agenesis in such conditions is important. Furthermore, the link between hypodontia and malocclusion was not examined in the present study.

To overcome these disadvantages, the study group should be as large as possible and a large amount of data should be analyzed. One strength of this study is its large sample size. Larger sample sizes have the obvious advantage of providing more data to determine the average values of the study population more accurately and to avoid errors from testing a small number of possibly atypical samples like those including only patients visiting orthodontic clinics.

We believe this type of study applying the TAC methodology (i.e., expressing both the number of missing teeth and their location by using a unique number) should be repeated at certain intervals in order to determine the homogenous patterns of anomalies in a certain population. Template treatment plans can be developed under the guidance of the results obtained upon extending such studies to many populations across the world. This, in turn, will lead to savings in terms of time and effort for dentists, while ensuring the patients receive optimum benefit from the treatments.

\section{CONCLUSION}

The prevalence of tooth agenesis was 7.25\%, and in most patients, two teeth were missing $(n=306 ; 42.7 \%)$. However, the difference in the prevalence of missing teeth per patient between the sexes was not statistically significant. The rate of agenesis as desbribed by five different patterns were $21.2 \%$ (q1) and 19.9\% (q2) in the maxillay arches while these rates were $23.9 \%$ (q3) and $23.3 \%$ (q4) in the mandibular arches. Investigation of these patterns revealed that the most common TAC value of the agenesis pattern in $q 1, q 2$, and $q 3$ was 16 (second premolars) and the most common pattern in q4 was 24 (both premolars). The present findings may be used to develop treatment protocols for the most common agenesis patterns and to increase the quality of interdisciplinary treatment for patients with hypodontia.

\section{CONFLICTS OF INTEREST}

No potential conflict of interest relevant to this article was reported.

\section{REFERENCES}

1. Nowak AJ, Christensen JR, Mabry TR, Townsend JA, Wells $\mathrm{MH}$. Pediatric dentistry: infancy through adolescence. Philadelphia: Elsevier; 2019.

2. Endo T, Ozoe R, Kubota M, Akiyama M, Shimooka S. A survey of hypodontia in Japanese orthodontic patients. Am J Orthod Dentofacial Orthop 2006;129:29-35.

3. Goya HA, Tanaka S, Maeda T, Akimoto Y. An orthopantomographic study of hypodontia in permanent teeth of Japanese pediatric patients. J Oral Sci 2008;50:143-50.

4. Tan SP, van Wijk AJ, Prahl-Andersen B. Severe hypodontia: identifying patterns of human tooth agenesis. Eur J Orthod 2011;33:150-4.

5. Arai K. Tooth agenesis patterns in Japanese orthodontic patients with nonsyndromic oligodontia. Am J Orthod Dentofacial Orthop 2019;156:238-47.

6. Káldy A, Balaton G. [Severe hypodontia in permanent dentition. Orthodontic treatment of oligodontia in children]. Fogorv Sz 2012;105:161-5. Hungarian.

7. Filius MAP, Cune MS, Créton M, Vissink A, Raghoebar GM, Visser A. Oral health-related quality of life in Dutch children diagnosed with oligodontia. A cross-sectional study. Int J Environ Res Public Health 2019;16:2371.

8. Locker D, Jokovic A, Prakash P, Tompson B. Oral health-related quality of life of children with oligodontia. Int J Paediatr Dent 2010;20:8-14. 
9. Silva Meza R. Radiographic assessment of congenitally missing teeth in orthodontic patients. Int $\mathrm{J}$ Paediatr Dent 2003;13:112-6.

10. lezzi G, Perrotti V, Piattelli A, D’Arcangelo C, Altieri F, Cassetta M. Split crest technique for implant treatment of agenesis of the upper lateral incisors: results of a randomized pilot histological and clinical study at 24-month follow-up. Braz Oral Res 2020;34:e118.

11. Sisman Y, Uysal T, Gelgor IE. Hypodontia. Does the prevalence and distribution pattern differ in orthodontic patients? Eur J Dent 2007;1:167-73.

12. Aren G, Guven Y, Guney Tolgay C, Ozcan I, Bayar $\mathrm{OF}$, Kose TE, et al. The prevalence of dental anomalies in a Turkish population. J Istanb Univ Fac Dent 2015;49:23-8.

13. Cantekin K, Dane A, Miloglu O, Kazanci F, Bayrakdar $\mathrm{S}$, Celikoglu M. Prevalence and intra-oral distribution of agenesis of permanent teeth among Eastern Turkish children. Eur J Paediatr Dent 2012;13:53-6.

14. Kirzioğlu Z, Köseler Sentut T, Ozay Ertürk MS, Karayilmaz $\mathrm{H}$. Clinical features of hypodontia and associated dental anomalies: a retrospective study. Oral Dis 2005;11:399-404.

15. Gökkaya B, Motro M, Kargül B. Prevalence and characteristics of non-syndromic hypodontia among Turkish orthodontic patient population. J Int Soc Prev Community Dent 2015;5:170-5.

16. van Wijk AJ, Tan SP. A numeric code for identifying patterns of human tooth agenesis: a new approach. Eur J Oral Sci 2006;114:97-101.

17. Souza-Silva BN, Vieira WA, Bernardino íM, Batista MJ, Bittencourt MAV, Paranhos LR. Non-syndromic tooth agenesis patterns and their association with other dental anomalies: a retrospective study. Arch Oral Biol 2018;96:26-32.

18. Gkantidis N, Katib H, Oeschger E, Karamolegkou M, Topouzelis N, Kanavakis G. Patterns of nonsyndromic permanent tooth agenesis in a large orthodontic population. Arch Oral Biol 2017;79:427.

19. Park MK, Shin MK, Kim SO, Lee HS, Lee JH, Jung $\mathrm{HS}$, et al. Prevalence of delayed tooth development and its relation to tooth agenesis in Korean children.
Arch Oral Biol 2017;73:243-7.

20. Lauwers L, Wojcik T, Delbarre A, Movaghar R, Ferri J. [Hypodontia: therapeutic strategy elaborated from 30 cases]. Rev Stomatol Chir Maxillofac 2009;110:263-8. French.

21. Nunn JH, Carter NE, Gillgrass TJ, Hobson RS, Jepson NJ, Meechan JG, et al. The interdisciplinary management of hypodontia: background and role of paediatric dentistry. Br Dent J 2003;194:245-51.

22. Ota S, Hirakata C, Endo T. Prevalence and patterns of tooth agenesis among malocclusion classes in a Japanese orthodontic population. J Oral Sci 2019;61:504-7.

23. Konstantonis D, Alexandropoulos A, Konstantoni N, Nassika M. A cross-sectional analysis of the prevalence of tooth agenesis and structural dental anomalies in association with cleft type in non-syndromic oral cleft patients. Prog Orthod 2017;18:20.

24. Al-Abdallah M, AlHadidi A, Hammad M, Al-Ahmad $H$, Saleh R. Prevalence and distribution of dental anomalies: a comparison between maxillary and mandibular tooth agenesis. Am J Orthod Dentofacial Orthop 2015;148:793-8.

25. Zhang J, Liu HC, Lyu X, Shen GH, Deng XX, Li WR, et al. Prevalence of tooth agenesis in adolescent Chinese populations with or without orthodontics. Chin J Dent Res 2015;18:59-65.

26. Bozga A, Stanciu RP, Mănuc D. A study of prevalence and distribution of tooth agenesis. $\mathrm{J}$ Med Life 2014;7:551-4.

27. Mani SA, Mohsin WS, John J. Prevalence and patterns of tooth agenesis among Malay children. Southeast Asian J Trop Med Public Health 2014;45: 490-8.

28. Guan G, Wang Y, Lo T, Preston B. Prevalence of tooth agenesis in orthodontic patient population in Western New York. N Y State Dent J 2013;79:31-5.

29. Celikoglu M, Kazanci F, Miloglu 0, Oztek 0, Kamak $\mathrm{H}$, Ceylan 1. Frequency and characteristics of tooth agenesis among an orthodontic patient population. Med Oral Patol Oral Cir Bucal 2010;15:e797-801.

30. Dang HQ, Constantine S, Anderson PJ. The prevalence of dental anomalies in an Australian population. Aust Dent J 2017;62:161-4. 